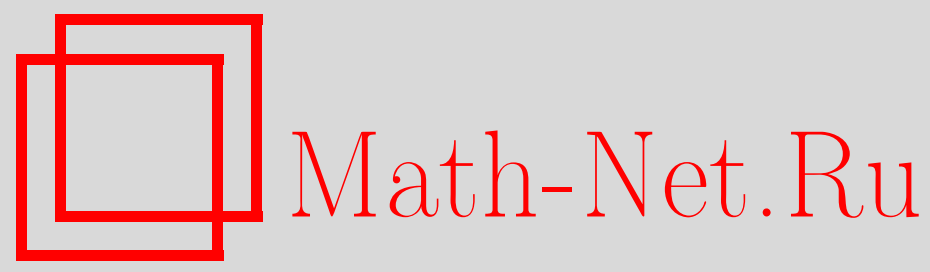

Л. А. Игнаточкина, В. Ф. Кириченко, Конформноинвариантные свойства приближенно келеровых многообразий, Матем. заметки, 1999, том 66, выпуск 5, 653-663

DOI: https://doi.org/10.4213/mzm1209

Использование Общероссийского математического портала Math-Net.Ru подразумевает, что вы прочитали и согласны с пользовательским соглашением http://www.mathnet.ru/rus/agreement

Параметры загрузки:

IP: 54.80 .73 .141

26 апреля 2023 г., 06:09:00

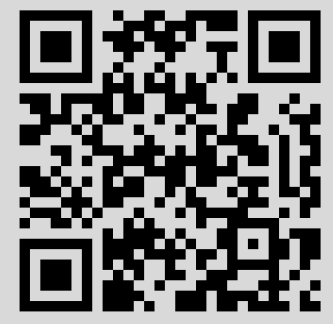




\title{
КОНФОРМНО-ИНВАРИАНТНЫЕ СВОЙСТВА ПРИБЛИЖЕННО КЕЛЕРОВЫХ МНОГООБРАЗИЙ
}

\author{
Л. А. Игнаточкина, В. Ф. Кириченко
}

\begin{abstract}
Доказано, что у метрики приближенно келеровых многообразий существует два нетривиальных основных конформньх инварианта. Доказано, что обращение в нуль одного из них равносильно конформной плоскости многообразия. Получено исчерпывающее описание приближенно келеровых многообразий с нулевым вторым конформным инвариантом. Результаты работы существенно обобщают известные результаты Ватанабэ и Такамацу, касающиеся классификации конформно-плоских приближенно келеровых многообразий.
\end{abstract}

Библиография: 19 названий.

Изучение конформно-инвариантных свойств римановых многообразий, в том числе и снабженных дополнительной структурой, занимает важное место в современных дифференциально-геометрических исследованиях. Сюда можно отнести, в частности, изучение конформно-инвариантных свойств почти эрмитовых многообразий, начатое в работе Грея и Хервеллы [1]. В этой работе авторы выделили классы почти эрмитовых структур, инвариантные относительно конформных преобразований метрики. Один из таких классов, класс $W_{4}$ в классификации Грея-Хервеллы, содержит структуры, локально конформные келеровой структуре и подробно изучавшиеся в работах Вайсмана [2], [3], Грея и Ванхекке [4], В.Ф. Кириченко [5], [6] и других авторов. Эти структуры представляют интерес не только с геометрической, но и с физической точки зрения [7]. Естественным расширением класса $W_{4}$ является класс $W_{1} \oplus W_{4}$ в классификации Грея-Хервеллы (класс структур Вайсмана-Грея [8]), содержаший структуры, локально конформные приближенно келеровым структурам (lcN $\mathscr{K}$-структуры). Однако геометрия этих структур пока изучена недостаточно. Поскольку в конформном классе метрики lcN $\mathscr{K}$-структуры содержится метрика, которая вместе с почти комплексной структурой многообразия образует приближенно келерову структуру, вопрос об изучении lcN $\mathscr{K}$-многообразий сводится к вопросу об изучении конформно-инвариантных свойств приближенно келеровых многообразий. Изучению этого вопроса и посвящена настоящая работа. В ней показано, что метрика приближенно келерова многообразия имеет два нетривиальных основных конформных инварианта. Обращениев нуль первого из них равносильно конформной плоскости многообразия. Поэтому наибольший интерес представляет второй инвариант. Получено исчерпывающее описание приближенно келеровых многообразий, для которых этот инвариант обращается в нуль: каждое такое многообразие является либо 2-мерным келеровым многообразием, либо 4-мерным келеровым многообразием нулевой скалярной кривизны, либо риччи-плоским келеровым многообразием размерности свьше четырех, либо шестимерным собственным приближенно келеровьм многообразием, либо произведением шестимерного собственного 
приближенно келерова многообразия и двумерного келерова многообразия постоянной отрицательной кривизны, причем справедливо и обратное.

Пусть $M$ - почти эрмитово многообразие с почти эрмитовой структурой $(J, g=\langle\cdot, \cdot\rangle)$, $J^{2}=-\mathrm{id},\langle J X, J Y\rangle=\langle X, Y\rangle, \operatorname{dim} M=2 n>2, \mathfrak{X}(M)$ - модуль гладких векторных полей на $M, \nabla$ - риманова связность на $M, d$ - оператор внешнего дифференцирования. Все многообразия, тензорные поля и т.п. объекты предполагаются гладкими класса $C^{\infty}$. Хорошо известно, что задание почти эрмитовой структуры на $M$ равносильно заданию $G$-структуры на $M$ со структурной группой $U(n)$, элементами пространства которой являются реперы, состояшие из попарно сопряженных собственных векторов структурного эндоморфизма $J$ и унитарные в естественной эрмитовой метрике комплексификации соответствующего касательного пространства (такие реперы называются $A$-реперами) [9]. Эта $G$-структура называется присоединенной. Будем предполагать, что индексы $i, j, k, \ldots$ пробегают значения от 1 до $2 n$, а индексы $a, b, c, d, f$, $g, h$ - значения от 1 до $n$. Положим $\hat{a}=a+n$. Как обычно, по индексам, заключенным в квадратные (соответственно круглые) скобки, подразумевается альтернирование (соответственно симметризация). Легко проверить, что на пространстве присоединенной $G$-структуры компоненты тензоров $g$ и $J$ задаются матрицами

$$
\left(g_{i j}\right)=\left(\begin{array}{cc}
0 & I_{n} \\
I_{n} & 0
\end{array}\right), \quad\left(J_{j}^{i}\right)=\left(\begin{array}{cc}
\sqrt{-1} I_{n} & 0 \\
0 & -\sqrt{-1} I_{n}
\end{array}\right),
$$

где $I_{n}$ - единичная матрица порядка $n$.

Напомним [1], [9], что почти эрмитова структура назьвается приближенно келеровой, (короче, N $\mathscr{K}$-)структурой, если ее фундаментальная форма $\Omega(X, Y)=\langle X, J Y\rangle$ является формой Киллинга или, что равносильно,

$$
\nabla_{X}(J) X=0, \quad X \in \mathfrak{X}(M) .
$$

Приближенно келерова структура назьвается келеровой, если $\nabla J=0$, и собственной в противном случае. Хорошо известно [9], что структурные уравнения римановой связности приближенно келеровой структуры на пространстве присоединенной $G$-структуры (назьваемые структурными уравнениями приближенно келеровой структуры) имеют вид:

1) $d \omega^{a}=\omega_{b}^{a} \wedge \omega^{b}+B^{a b c} \omega_{b} \wedge \omega_{c}$

2) $d \omega_{a}=-\omega_{a}^{b} \wedge \omega_{b}+B_{a b c} \omega^{b} \wedge \omega^{c}$

3) $d \omega_{b}^{a}=\omega_{c}^{a} \wedge \omega_{b}^{c}+\left(2 B^{a d h} B_{h b c}+A_{b c}^{a d}\right) \omega^{c} \wedge \omega_{d}$,

где $\omega_{a}=\omega^{\hat{a}},\left\{B^{a b c}\right\},\left\{B_{a b c}\right\}$ и $\left\{A_{b c}^{a d}\right\}$ - системы функций на пространстве присоединенной $G$-структуры, служашие компонентами (комплексных) чистых тензоров на $M$, назьваемых структурными тензорами первого, второго и третьего рода, соответственно. При этом структурные тензоры первого и второго рода кососимметричны, а структурньй тензор третьего рода симметричен по любой паре верхних либо нижних индексов [9]. Кроме того, известно, что компоненты структурных тензоров первого и второго рода удовлетворяют тождествам

1) $d B^{a b c}-B^{h b c} \omega_{h}^{a}-B^{a h c} \omega_{h}^{b}-B^{a b h} \omega_{c}^{h}=0$,

2) $d B_{a b c}+B_{h b c} \omega_{a}^{h}+B_{a h c} \omega_{b}^{h}+B_{a b h} \omega_{h}^{c}=0$, 
что равносильно параллельности этих тензоров в присоединенной связности [10], а компоненты структурного тензора третьего рода удовлетворяют тождеству

$$
d A_{b c}^{a d}+A_{h c}^{a d} \omega_{b}^{h}+A_{b h}^{a d} \omega_{c}^{h}-A_{b c}^{h d} \omega_{h}^{a}-A_{b c}^{a h} \omega_{h}^{d}=A_{b c h}^{a d} \omega^{h}+A_{b c}^{a d h} \omega_{h},
$$

где $\left\{A_{b c h}^{a d}\right\}$ и $\left\{A_{b c}^{a d h}\right\}$ - системы функций на пространствеприсоединенной $G$-структуры, симметричных по любой паре верхних либо нижних индексов и служащих компонентами ковариантного дифференциала структурного тензора третьего рода в присоединенной связности. (Напомним, что присоединенной связностью (обобщенного) почти эрмитова многообразия называется связность $\widetilde{\nabla}=\nabla+T$, где $T$ - композиционный тензор присоединенной $Q$-алгебры [11]). Напомним также [9], что компоненты структурных тензоров удовлетворяют соотношениям комплексной сопряженности

$$
\bar{B}^{a b c}=B_{a b c}, \quad \bar{A}_{b c}^{a d}=A_{a d}^{b c} .
$$

Кроме того, согласно [12] имеет место тождество

$$
A_{a f}^{a c} B_{g d c}-A_{a g}^{a c} B_{f d c}=4 B^{a h c} B_{a h d} B_{g f c} .
$$

Примем для удобства следующие обозначения:

$$
B_{b c}^{a d}=B^{a d h} B_{h b c}, \quad B_{b}^{a}=B_{b c}^{a c}, \quad A_{b}^{a}=A_{b c}^{a c}, \quad A_{c}^{a b}=A_{h c}^{h a b}, \quad A_{a b}^{c}=A_{h a b}^{h c} .
$$

Лемма 1. Пусть $M-\mathrm{N} \mathscr{K}$-многообразие. Тогда $B=B_{h}^{h}$ - неотрицательная константа. При этом $B=0$ тогда и только тогда, когда $M$ - келерово многообразие.

ДоКАЗАТЕЛЬСТВо. Согласно определению и (4)

$$
B=B^{a b c} B_{a b c}=\sum_{a b c}\left|B_{a b c}\right|^{2} \geqslant 0, \text { причем } B=0 \Longleftrightarrow B_{a b c}=B^{a b c}=0 .
$$

Остается напомнить, что структурные тензоры первого и второго рода $\mathrm{N} \mathscr{K}$-многообразия - нулевые тогда и только тогда, когда это многообразие является келеровым [13]. Наконец, дифференцируя тождество $B^{a b c} B_{a b c}=B$ внешним образом, с учетом (2) получаем, что $d B=0$, и значит, $B=$ const.

Пусть $R, r$ и $\varkappa$ - тензор Римана-Кристоффеля, тензор Риччи и скалярная кривизна метрики $\langle\cdot, \cdot\rangle \mathrm{N} \mathscr{K}$-многообразия $M$ соответственно. С учетом (1) нетрудно вычислить выражение компонент тензора $R$ на пространстве присоединенной $G$-структуры (т.е. в $A$-репере):

$$
R_{\hat{a} b c \hat{d}}=B_{b c}^{a d}+A_{b c}^{a d}, \quad R_{\hat{a} \hat{b} c d}=-2 B_{c d}^{a b},
$$

плюс соотношения, полученные с учетом классических свойств симметрии этого тензоpa. Остальные его компоненты равны нулю. С учетом этих соотношений нетрудно найти выражение компонент тензора $r$ и скалярной кривизны $\varkappa$ многообразия $M$ в $A$-репере:
1) $r_{\hat{a} b}=r_{b \hat{a}}=3 B_{b}^{a}-A_{b}^{a}$,
2) $r_{a b}=r_{\hat{a} \hat{b}}=0$,
3) $\varkappa=6 B-2 A_{c}^{c}$.

Теперь нетрудно найти выражение компонент основного конформного инварианта риманова многообразия - тензора Вейля $C$ конформной кривизны. Этот тензор канонически 
представляется в виде суммы восьми элементов своего спектра, т.е. тензоров того же типа $(3,1)$, комплексно линейных либо комплексно антилинейных по различньм аргументам [11]. В силу конформной инвариантности структурного эндоморфизма $J$ эти тензоры также конформно инвариантны, и их совокупность образует систему основных конформных инвариантов почти эрмитова многообразия. Покажем, что N $\mathscr{K}$-многообразие имеет не более двух (с точностью до свойств симметрии тензора Вейля) ненулевых основных конформных инварианта. Напомним [14], что тензор $C$ многообразия $M^{2 n}$ определяется формулой

$$
C_{i j k l}=R_{i j k l}+\frac{1}{2(n-1)}\left(r_{i k} g_{j l}+r_{j l} g_{i k}-r_{i l} g_{j k}-r_{j k} g_{i l}\right)+\frac{\varkappa\left(g_{j k} g_{i l}-g_{j l} g_{i k}\right)}{(2 n-1)(2 n-2)} .
$$

С учетом (6) находим, что на пространстве присоединенной $G$-структуры эти соотношения принимают вид:

$$
\begin{aligned}
& \text { 1) } C_{\hat{a} b c \hat{d}}=B_{b c}^{a d}+A_{b c}^{a d}-\frac{1}{2(n-1)}\left(r_{c}^{a} \delta_{b}^{d}+r_{b}^{d} \delta_{c}^{a}\right)+\frac{\varkappa}{(2 n-1)(2 n-2)} \delta_{c}^{a} \delta_{b}^{d}, \\
& \text { 2) } C_{\hat{a} \hat{b} c d}=-2 B_{c d}^{a b}+\frac{2}{n-1} r_{[c}^{[a} \delta_{d]}^{b]}-\frac{\varkappa}{(2 n-1)(2 n-2)} \delta_{c d}^{a b}
\end{aligned}
$$

(где $\delta_{c d}^{a b}=\delta_{c}^{a} \delta_{d}^{b}-\delta_{d}^{a} \delta_{c}^{b}$ ), плюс соотношения, полученные с учетом классических свойств симметрии этого тензора, аналогичных свойствам симметрии тензора $R$. Именно эти выражения (и им сопряженные) и задают компоненты основных конформных инвариантов $\mathrm{N} \mathscr{K}$-многообразия.

ЗАмЕчаниЕ 1. Очевидно, остальные компоненты тензора Вейля, а значит, и остальные основные конформные инварианты $\mathrm{N} \mathscr{K}$-многообразия равны нулю.

Исследуем геометрический смысл обращения в нуль основных конформных инвариантов.

В силу тождества Риччи (верного, как хорошо известно, и для тензора Вейля) обращение в нуль инварианта $\mathscr{C}_{1}=\left\{C_{\hat{a} b c \hat{d}}\right\}$ влечет обращение в нуль инварианта $\mathscr{C}_{2}=$ $\left\{C_{\hat{a} \hat{b} c d}\right\}$ и, следовательно, обращение в нуль тензора Вейля, т.е. конформную плоскость многообразия. Поэтому самостоятельньй интерес имеет рассмотрение именно инварианта $\mathscr{C}_{2}$.

ПРЕДЛОЖЕНИЕ 1. Конформный инвариант $\mathscr{C}_{2}$ вычисляется по формуле

$$
\mathscr{C}_{2}(X, Y) Z=\frac{1}{2}(C(X, Y) Z-C(J X, J Y) Z), \quad X, Y, Z \in \mathfrak{X}(M) .
$$

ДокАЗАТЕЛЬСтво. С учетом замечания 1 имеем:

$$
(C(X, Y) Z)^{a}=C^{a}{ }_{i j k} X^{j} Y^{k} Z^{i}=C_{\hat{a} \hat{b} c d} X^{c} Y^{d} Z^{\hat{b}}+C_{\hat{a} b \hat{c} d} X^{\hat{c}} Y^{d} Z^{b}+C_{\hat{a} b c \hat{d}} X^{c} Y^{\hat{d}} Z^{b} .
$$

Проводя аналогичное вычисление для тензора $C(J X, J Y) Z$ с учетом того, что в $A$-репере справедливы соотношения $J_{b}^{a}=i \delta_{b}^{a}, J_{\hat{b}}^{\hat{a}}=-i \delta_{a}^{b}$, и почленно вычитая найденные выражения, получим, что

$$
(C(X, Y) Z-C(J X, J Y) Z)^{a}=2 C_{\hat{a} \hat{b} c d} X^{c} Y^{d} Z^{\hat{b}} .
$$

Таким образом, функции $C_{\hat{a} \hat{b} c d}$ и им комплексно сопряженные являются компонентами тензора $\frac{1}{2}(C(X, Y) Z-C(J X, J Y) Z)$, а следовательно, этот тензор совпадает с тензором $\mathscr{C}_{2}(X, Y) Z$. 
ОПРЕДЕЛЕНИЕ 1. Приближенно келерово многообразие, для которого $\mathscr{C}_{2}=0$, назовем $\mathrm{N} \mathscr{K}$-многообразием класса $\mathscr{C}_{2}$ или конформно-паракелеровым многообразием. В силу предложения 1 , такое $\mathrm{N} \mathscr{K}$-многообразие определяется тождеством

$$
C(J X, J Y) Z=C(X, Y) Z, \quad X, Y, Z \in \mathfrak{X}(M) .
$$

Пусть $M$ - конформно-паракелерово многообразие. В силу 2) из (8) это равносильно тому, что

$$
B_{c d}^{a b}=\frac{1}{4(n-1)}\left(r_{c}^{a} \delta_{d}^{b}+r_{d}^{b} \delta_{c}^{a}-r_{d}^{a} \delta_{c}^{b}-r_{c}^{b} \delta_{d}^{a}\right)-\frac{\varkappa}{2(2 n-1)(2 n-2)} \delta_{c d}^{a b} .
$$

Сворачивая это соотношение сначала по индексам $b$ и $d$, а затем по индексам $a$ и $c$, получим, что

$$
B=\frac{n-1}{4(2 n-1)} \varkappa .
$$

С учетом леммы 1 получаем следующий результат.

Теорема 1. Конформно-паракелерово многообразие $M$ является римановым многообразием постоянной неотрицательной скалярной кривизны. При этом его скалярная кривизна равна нулю тогда и только тогда, когда $M$ - келерово многообразие.

Найдем теперь аналитический критерий принадлежности $\mathrm{N} \mathscr{K}$-многообразия $M$ классу $\mathscr{C}_{2}$ в терминах структурных тензоров.

ТЕОрема 2. Приближенно келерово многообразие $M$ размерности свыше четырех является конформно-паракелеровым тогда и только тогда, когда на пространстве присоединенной G-структурь

$$
\text { 1) } B_{b c}^{a d}=t_{[b}^{[a} \delta_{c]}^{d]}, \quad \text { 2) } \quad r_{b}^{a}=\frac{4(n-1)}{n-2} B_{b}^{a}-\frac{2}{(n-1)(n-2)} B \delta_{b}^{a}
$$

где $t$ - подходящий тензор типа $(1,1)$ на $M$, причем с необходимостью

$$
t_{b}^{a}=\frac{4}{n-2} B_{b}^{a}-\frac{2}{(n-1)(n-2)} B \delta_{b}^{a}
$$

Для такого многообразия

$$
A_{b}^{a}=-\frac{n+2}{n-2} B_{b}^{a}+\frac{2}{(n-1)(n-2)} B \delta_{b}^{a} .
$$

ДокАЗАТЕЛЬСТво. Пусть $M$-конформно-паракелеровомногообразиеразмерности свьше четырех. Сворачивая (10) по индексам $b$ и $d$, выразим отсюда $r_{c}^{a}$ :

$$
r_{c}^{a}=\frac{4(n-1)}{n-2} B_{c}^{a}-\frac{1}{2(n-2)(2 n-1)} \varkappa \delta_{c}^{a} .
$$

С учетом (11) отсюда получается 2) из (12). Подставляя его и (11) в (10), получим, что

$$
B_{c d}^{a b}=\frac{4}{n-2} B_{[c}^{[a} \delta_{d]}^{b]}-\frac{1}{(n-1)(n-2)} B \delta_{c d}^{a b} .
$$


Очевидно, это соотношение можно записать в форме (12), приняв за $t_{b}^{a}$ выражение (13). Более того, последовательно свертьвая 1) из (12) сначала по индексам $a$ и $c$, а потом по индексам $b$ и $d$, легко получить, что (13) с необходимостью следует из (12). Наконец, (14) непосредственно следует из (7) и (12).

Обратно, пусть $M$ - N $\mathscr{K}$-многообразие размерности свыше четырех, на котором выполняются тождества (12). Как уже отмечалось, (13) с необходимостью следует из (12). Кроме того, сворачивая 2) из (12), получим соотношение (11). Теперь непосредственная проверка показывает, что (10) вьполняется тождественно в силу (11), (12) и (13). Следовательно, $M$ - конформно-паракелерово многообразие.

СлЕДСТВИЕ. Келерово многообразие размерности свыше четырех конформнопаракелерово тогда и только тогда, когда оно риччи-плоско.

ДокАЗАТЕЛЬСТво. Очевидно, для келеровых многообразий соотношение 1) из (12) выполняется тождественно. Соотношение же 2) из (12) для них принимает вид $r_{b}^{a}=0$, что ввиду (7) равносильно тому, что многообразие риччи-плоско.

Теорема 3. Четырехмерное приближенно келерово многообразие $M$ является конформно-паракелеровым тогда и только тогда, когда выполняется одно из следующих равносильных условий:

$$
\text { 1) } \left.A_{c}^{c}=0, \quad 2\right) \quad \varkappa=0 \text {. }
$$

ДокАЗАТЕЛЬСтво. Прежде всего, структурные тензоры четырехмерного N $\mathscr{K}$-многообразия в силу своей кососимметричности нулевые и в силу леммы 1 такое многообразие - келерово. Но тогда с учетом (7) $r_{c}^{a}=-A_{c}^{a}$, в силу чего (а также в силу леммы 1 ) соотношение $(10)$, равносильное обрашению в нуль инварианта $\mathscr{C}_{2}$, примет вид:

$$
A_{c}^{a} \delta_{b}^{d}+A_{b}^{d} \delta_{c}^{a}-A_{b}^{a} \delta_{c}^{d}-A_{c}^{d} \delta_{b}^{a}=0 .
$$

Поскольку все индексы в этом соотношении пробегают лиш два значения, непосредственная проверка показывает, что оно равносильно условию 1) из (15). С другой стороны, соотношение 3) из (7) показьвает, что в случае келерова многообразия соотношения 1) и 2) из (15) равносильны.

С учетом теоремы 4 в [15] немедленно получаем

СлЕДСТВИЕ. Четырехмерное келерово многообразие является конформно-паракелеровым тогда и только тогда, когда оно антиавтодуально.

Пусть $M$ - конформно-плоское N $\mathscr{K}$-многообразие. Как уже отмечалось, это равносильно обращению в нуль инварианта $\mathscr{C}_{1}$, что в силу 1) из (8) равносильно соотношению

$$
B_{b c}^{a d}+A_{b c}^{a d}=\frac{1}{2(n-1)}\left(r_{c}^{a} \delta_{b}^{d}+r_{b}^{d} \delta_{c}^{a}\right)+\frac{\varkappa}{(2 n-1)(2 n-2)} \delta_{c}^{a} \delta_{b}^{d} .
$$

Альтернируя и симметризуя это соотношение по индексам $a$ и $d$, получим соответственHO:

$$
\begin{aligned}
B_{b c}^{a d} & =\frac{1}{n-1} r_{[b}^{[a} \delta_{c]}^{d]}-\frac{\varkappa}{2(2 n-1)(2 n-2)} \delta_{b c}^{a d}, \\
A_{b c}^{a d} & =-\frac{1}{n-1} r_{(b}^{(a} \delta_{c)}^{d)}+\frac{\varkappa}{2(2 n-1)(2 n-2)} \tilde{\delta}_{b c}^{a d},
\end{aligned}
$$

где $\tilde{\delta}_{b c}^{a d}=\delta_{b}^{a} \delta_{c}^{d}+\delta_{c}^{a} \delta_{b}^{d}$. Далее, рассуждая так же, как и при доказательстве теорем 2 и 3 , получаем следующий результат. 
ТЕоремА 4. Приближенно келерово многообразие М размерности свыше четырех является конформно-плоским тогда и только тогда, когда на пространстве присоединенной G-структуры

$$
B_{b c}^{a d}=t_{[b}^{[a} \delta_{c]}^{d]}, \quad A_{b c}^{a d}=-t_{(b}^{(a} \delta_{c)}^{d)},
$$

где $t$ - подходящий тензор типа $(1,1)$ на $M$, причем с необходимостью

$$
t_{b}^{a}=\frac{4}{n-2} B_{b}^{a}-\frac{2}{(n-1)(n-2)} B \delta_{b}^{a} .
$$

СлЕДСТВИЕ. Конформно-плоское келерово многообразие размерности свыше четырех локально голоморфно изометрично многообразию $\mathbb{C}^{n}$, снабжсенному канонической келеровой структурой.

ДокАЗАТЕЛЬСТво. Это непосредственно следует из того, что в силу теоремы 4 для такого многообразия $A_{b c}^{a d}=0$, а в силу соотношений (6) такое многообразие локально плоско, в частности, является комплексной пространственной формой нулевой голоморфной секционной кривизны, а значит, локально голоморфно изометрично $\mathbb{C}^{n}$.

ТЕОРема 5. Четырехмерное приближенно келерово многообразие является конформно-плоским тогда и только тогда, когда

$$
A_{b c}^{a d}=\frac{1}{2}\left(A_{c}^{a} \delta_{b}^{d}+A_{b}^{d} \delta_{c}^{a}\right) .
$$

ДокАЗАТЕЛЬСтво. Пусть $M-4$-мерное конформно-плоское келерово многообразие. В силу теоремы 1 его скалярная кривизна равна нулю, а в силу соотношений (7) имеем: $r_{b}^{a}=-A_{b}^{a}$. Поэтому соотношения (16) примут вид:

$$
\begin{aligned}
& \text { 1) } A_{c}^{a} \delta_{b}^{d}+A_{b}^{d} \delta_{c}^{a}-A_{b}^{a} \delta_{c}^{d}-A_{c}^{d} \delta_{b}^{a}=0 \\
& \text { 2) } A_{b c}^{a d}=\frac{1}{4}\left(A_{c}^{a} \delta_{b}^{d}+A_{b}^{d} \delta_{c}^{a}+A_{b}^{a} \delta_{c}^{d}+A_{c}^{d} \delta_{b}^{a}\right) .
\end{aligned}
$$

Подставляя (17a) в $(17 \mathrm{~b})$, получим, что $A_{b c}^{a d}=\frac{1}{2}\left(A_{c}^{a} \delta_{b}^{d}+A_{b}^{d} \delta_{c}^{a}\right)$. Обратно, пусть для 4 -мерного келерова многообразия $M$ имеет место это соотношение. Свертывая его по индексам $d$ и $b$, получим, что $A_{c}^{c}=0$ и в силу (7), $=0$. С другой стороны, в силу свойств симметрии третьего структурного тензора это соотношение влечет (17a), a значит, и (17b). Следовательно, многообразие $M$ конформно-плоско.

Вернемся к рассмотрению конформно-паракелеровых $\mathrm{N} \mathscr{K}$-многообразий.

Теорема 6. Приближенно келерово многообразие $M$ конформно-паракелерово тогда и только тогда, когда оно является многообразием одного из следующих классов:

1) риччи-плоским келеровым многообразием размерности свыше четырех;

2) 4-мерным келеровым многообразием нулевой скалярной кривизны;

3) 2-мерным келеровым многообразием;

4) 6-мерным собственным приближенно келеровым многообразием;

5) многообразием, локально голоморфно изометричным произведению 6-мерного собственного приближенно келерова многообразия и 2-мерного келерова многообразия постоянной отричательной кривизны $-\frac{1}{3} B$. 
ДокАЗАТЕЛЬСТво. Если $M$ - келерово многообразие размерности свьше четырех, утверждение теоремы совпадает со следствием теоремы 2 . Если $M-4$-мерное келерово многообразие, утверждение теоремы совпадает с утверждением теоремы 3 . Если $M-$ двумерное келерово многообразие, то хорошо известно, что оно (локально) конформно-плоско, что выражается в существовании на нем атласа, состоящего из изотермических координатных карт. В частности, такое многообразие конформно-паракелерово. Таким образом, теорема справедлива в случае келеровых многообразий.

Пусть теперь $M-$ собственное $\mathrm{N} \mathscr{K}$-многообразие (с необходимостью размерности свьше четырех). Заметим, что эндоморфизм $\mathfrak{b}$, задаваемый в $A$-репере матрицей $\left(B_{b}^{a}\right)$, эрмитово-симметричен в силу (4) и, значит, диагонализуем в подходящем $A$-репере, т.е. в этом репере

$$
B_{b}^{a}=B_{b} \delta_{b}^{a},
$$

где $\left\{B_{a}\right\}$ - собственные значения этого эндоморфизма. Более того, эрмитова форма $\mathfrak{B}(X, Y)=B_{b}^{a} X^{b} Y_{a}$, отвечающая этому эндоморфизму, положительно полуопределена, поскольку

$$
\mathfrak{B}(X, X)=B_{b}^{a} X^{b} X_{a}=B^{c d a} B_{c d b} X^{b} X_{a}=\sum_{c, d}\left|B_{c d a} X^{a}\right|^{2} \geqslant 0 .
$$

Следовательно, $B_{a} \geqslant 0, a=1, \ldots, n$. Если $M$ - конформно-паракелерово многообразие, то подставляя (14) и (18) в (5), получим, что

$$
\left(\frac{n+2}{n-2}\left(B_{g}+B_{f}\right)-4 B_{d}-\frac{4}{(n-1)(n-2)} B\right) B_{f d g}=0 .
$$

Поскольку $M$ - собственное $\mathrm{N} \mathscr{K}$-многообразие, его структурные тензоры первого и второго рода ненулевые, и значит, существует тройка индексов $f, d$ и $g$ такая, что $B_{f d g} \neq 0$. Для нее соотношение (19) можно записать в виде системы

$$
\begin{aligned}
& (n+2)\left(B_{g}+B_{f}\right)-4(n-2) B_{d}=\frac{4}{n-1} B, \\
& (n+2)\left(B_{d}+B_{g}\right)-4(n-2) B_{f}=\frac{4}{n-1} B, \\
& (n+2)\left(B_{f}+B_{d}\right)-4(n-2) B_{g}=\frac{4}{n-1} B .
\end{aligned}
$$

Поскольку эта система имеет ненулевой детерминант, система имеет единственное решение, и так как неизвестные $B_{d}, B_{f}$ и $B_{g}$ входят в систему симметрично, $B_{d}=B_{f}=$ $B_{g}$, и в силу уравнений системы

$$
(6-n) B_{d}=\frac{2}{n-1} B .
$$

Выражение в правой части этого тождества положительно, а значит, оно положительно и в его левой части и в силу сделанного вьше замечания $6-n>0$, т.е. $n<6$. C другой стороны, поскольку $M-$ собственное $\mathrm{N} \mathscr{K}$-многообразие, $n>2$, и значит, $n=3$ либо $n=4$, либо $n=5$. Заметим, что третий случай невозможен, поскольку в нем (20) принимает вид $2 B_{d}=B$, что противоречит тому, что $B=\sum_{d=1}^{n} B_{d} \geqslant 3 B_{d}$. Далее, в первом случае $M-6$-мерное собственное $\mathrm{N} \mathscr{K}$-многообразие. Во втором случае согласно [16] $M$ локально голоморфно изометрично произведению 6-мерного собственного N $\mathscr{K}$-многообразия $M_{1}$ на двумерное келерово многообразие $M_{2}$. Тогда модуль $\mathfrak{X}(M)$ 
распадается в прямую сумму распределений $\mathfrak{D}_{1}$ и $\mathfrak{D}_{2}$ размерностей 6 и 2 соответственно, инвариантных относительно структурного эндоморфизма и параллельных в римановой связности. Интегральные многообразия этих распределений локально голоморфно изометричны многообразиям $M_{1}$ и $M_{2}$ соответственно [16]. Рассмотрим редукцию присоединенной $G$-структуры по подгруппе $U(3) \times U(1)$, тотальное пространство которой состоит из $A$-реперов, адаптированных указанному разложению модуля $\mathfrak{X}(M)$. В силу (14) в таком $A$-репере $A_{4}^{4}=(1 / 3) B \delta_{4}^{4}$ и в силу (6)

$$
R_{4 \hat{4} 4 \hat{4}}=-A_{44}^{44}=-A_{4 c}^{4 c}=-A_{4}^{4}=-\frac{1}{3} B=-\frac{1}{3} B\left(g_{4 \hat{4}} g_{4 \hat{4}}-g_{44} g_{\hat{4} \hat{4}}\right) \text {. }
$$

Это означает, что $M_{2}$ - многообразие постоянной отрицательной кривизны $k=-B / 3$.

Обратно, если собственное $\mathrm{N} \mathscr{K}$-многообразие $M$ шестимерно, то оно является $\mathrm{N} \mathscr{K}$-многообразием ненулевого постоянного типа $B / 6$ и, следовательно, $B_{b c}^{a d}=$ $(B / 6) \delta_{b c}^{a d}$ (см. [13]). Но тогда $B_{b}^{a}=(1 / 3) B \delta_{b}^{a}$ и в силу (13) $t_{b}^{a}=(1 / 3) B \delta_{b}^{a}$, а в силу формулы (12) в [13] $A_{b}^{a}=-2 B_{b}^{a} / 3$. Наконец, в силу (7) $r_{b}^{a}=(5 / 3) B \delta_{b}^{a}$, откуда следует, что соотношения (12) выполняются тождественно, и значит, $M$ - конформно-паракелерово многообразие.

Пусть, наконец, собственное $\mathrm{N} \mathscr{K}$-многообразие $M$ восьмимерно. Как уже упоминалось, в этом случае согласно [16] $M$ локально голоморфно изометрично произведению 6-мерного собственного $\mathrm{N} \mathscr{K}$-многообразия $M_{1}$ на двумерное келерово многообразие $M_{2}$. Как и вьше, ограничимся рассмотрением $A$-реперов такого многообразия, адаптированных разложению $\mathfrak{X}(M)=\mathfrak{D}_{1} \oplus \mathfrak{D}_{2}$ в принятых вьше обозначениях. Пусть греческие индексы пробегают значения от 1 до 3. Тогда, как и выше,

$$
B_{\gamma \delta}^{\alpha \beta}=\frac{1}{6} B \delta_{\gamma \delta}^{\alpha \beta}, \quad B_{\beta}^{\alpha}=\frac{1}{3} B \delta_{\beta}^{\alpha}, \quad B_{4}^{4}=0
$$

и для тензора $t$, построенного согласно (13), имеем:

$$
t_{\beta}^{\alpha}=\frac{1}{3} B \delta_{\beta}^{\alpha}, \quad t_{4}^{4}=-\frac{1}{3} B
$$

Кроме того, согласно формуле $(12)$ в [13] $A_{\beta}^{\alpha}=-(2 / 3) B \delta_{\beta}^{\alpha}$. Наконец, по предположению $M_{2}$ - многообразие постоянной кривизны $k=-B / 3$ и в силу (6)

$$
A_{4}^{4}=A_{4 c}^{4 c}=A_{44}^{44}=-R_{4 \hat{4} 4 \hat{4}}=\frac{1}{3} B\left(g_{4 \hat{4}} g_{4 \hat{4}}-g_{44} g_{\hat{4} \hat{4}}\right)=\frac{1}{3} B
$$

Согласно (7) $r_{\beta}^{\alpha}=(5 / 3) B \delta_{\beta}^{\alpha}, r_{4}^{4}=-(1 / 3) B$. Теперь справедливость соотношений (12) для $M$ проверяется непосредственно. Следовательно, $M$ - конформно-паракелерово многообразие.

ЗАмЕчАниЕ 2. Легко видеть, что эндоморфизм b определен внутренним образом как сужение комплексификации $K$-оператора k присоединенной $K$-алгебры на собственные распределения структурного эндоморфизма $J$ (см. [17]).

В заключение покажем, как, используя теорему 6 , несложно получить полную классификацию конформно-плоских $\mathrm{N} \mathscr{K}$-многообразий. 
ТЕорема 7. Приближенно келерово многообразие $M$ конформно-плоско тогда $и$ только тогда, когда оно локально голоморфно изометрично одному из следующих многообразий:

1) комплексному евклидову пространству $\mathbb{C}^{n}$;

2) многообразию $S_{k}^{2} \times S_{-k}^{2}, k>0$;

3) 2-мерному келерову многообразию;

4) многообразию $S_{k}^{6}, \quad k>0$;

5) многообразию $S_{k}^{6} \times S_{-k}^{2}, k>0$, снабженных канонической $\mathrm{N} \mathscr{K}$-структурой. Здесь символ $S_{k}^{m}$ обозначает

ДокАЗАТЕЛЬСТво. Пусть $M$ - конформно-плоское $\mathrm{N} \mathscr{K}$-многообразие. Тогда оно, в частности, конформно-паракелерово, и следовательно, к нему применима теорема 6. Если $M$ - келерово многообразие размерности свьше четырех, утверждение теоремы 7 вытекает из следствия к теореме 4 . Если $M$ - конформно-плоское четырехмерное келерово многообразие, то оно, в частности, является автодуальным многообразием постоянной (нулевой) скалярной кривизны и согласно теореме 3 в [15] $M$ локально голоморфно изометрично многообразию вида $S_{k}^{2} \times S_{-k}^{2}$. Если $M$ - двумерное келерово многообразие, то, как уже упоминалось, оно всегда конформно-плоско. Если $M$ - шестимерное собственное $\mathrm{N} \mathscr{K}$-многообразие, то согласно теореме 3 в [13] оно является многообразием Эйнштейна с космологической константой $\varepsilon=5 B / 3$ и, значит,

$$
0 \equiv C_{i j k l}=R_{i j k l}+\frac{1}{3} B\left(g_{i k} g_{j l}-g_{i l} g_{j k}\right)
$$

т.е. $M-$ многообразие постоянной кривизны $B / 3$, в частности, N $\mathscr{K}$-многообразие постоянной голоморфной кривизны, и в силу [12] локально голоморфно изометрично многообразию вида $S_{k}^{6}, k>0$. Наконец, если $M$ - конформно-плоское восьмимерное собственное N $\mathscr{K}$-многообразие, то его шестимерная составляющая, очевидно, также конформно-плоская и по доказанному локально голоморфно изометрична многообразию вида $S_{k}^{6}, k>0$, где $k=B / 3$. В то же время, согласно теореме 6 его двумерная составляющая локально голоморфно изометрична многообразию вида $S_{-k}^{2}$.

Обратно, как хорошо известно [18], многообразия каждого из пяти типов, перечисленных в теореме 7, конформно плоски.

Заметим, что результат, полученньй в теореме 7 , из совершенно других соображений был получен Ватанабэ и Такамацу [19]. Таким образом, теорему 6 можно рассматривать как существенное обобщение результатов этих авторов.

\section{СПИСОК ЦИТИРОВАННОЙ ЛИТЕРАТУРЫ}

[1] Gray A., Hervella L. M. The sixteen classes of almost Hermitian manifolds and their linear invariants // Ann. Math. Pure Appl. 1980. V. 123. P. 35-58.

[2] Vaisman I. On locally and globally conformal Kähler manifold // Trans. Amer. Math. Soc. 1980. V. 262. P. 533-542.

[3] Vaisman I. Some curvature properties of locally conformal Kähler manifolds // Trans. Amer. Math. Soc. 1980. V. 259. P. 439-447.

[4] Gray A., Vanhecke L. Almost Hermitian manifolds with constant holomorphic sectional curvature // Cas. Pestov. Mat. 1979. V. 104. P. 170-179.

[5] Кириченко В.Ф. Локально конформно-келеровы многообразия постоянной голоморфной секционной кривизны // Матем. сб. 1991. Т. 182. № 3. С. 354-363.

[6] Кириченко В. Ф. Конформно плоские локально конформно-келеровы многообразия // Матем. заметки. 1992. Т. 51. № 5. С. 57-66. 
[7] Janus S., Visinescu M. Kaluza-Klein theory with scalar fields on generalized Hopf manifolds // Clas. Quantum Grav. 1987. V. 4. P. 1317-1325.

[8] Кириченко В. Ф., Ежова Н. А. Конформные инварианты многообразий Вайсмана-Грея // УМН. 1996. Т. 51. № 2. С. 163-164.

[9] Кириченко В.Ф. Дифференциальная геометрия $K$-пространств. // Итоги науки и техн. Т. 8. Проблемы геометрии. М.: ВИНИТИ АН СССР, 1977. С. 139-161.

[10] Кириченко В.Ф. К-пространства максимального ранга // Матем. заметки. 1977. Т. 22. № 3. C. $465-476$.

[11] Кириченко В.Ф. Методы обобщенной эрмитовой геометрии в теории почти контактных многообразий // Итоги науки и техники. Проблемы геометрии. Т. 18. М.: ВИНИТИ АН CCCP, 1986. C. $25-72$.

[12] Кириченко В.Ф. $K$-пространства постоянной голоморфной секционной кривизны // Матем. заметки. 1976. Т. 19. №6. С. 805-814.

[13] Кириченко В.Ф. K-пространства постоянного типа // Сиб. матем. ж. 1976. Т. 17. № 2. C. $282-289$.

[14] Рашевский П. К. Риманова геометрия и тензорный анализ. М.: Наука, 1976.

[15] Арсеньева О.Е. Автодуальная геометрия обобщенных келеровых многообразий // Матем. сб. 1993. Т. 184. № 2. С. 137-148.

[16] Кириченко В.Ф. Голоморфные подмногообразия обобщенных приближенно келеровых многообразий // Изв. ВУЗов. Матем. 1986. № 1. С. 43-48.

[17] Kirichenko V.F. Generalized quasi-Kaehlerian manifolds and axioms of $C R$-submanifolds in generalized Hermitian geometry, II // Geom. Dedicata. 1994. V. 51. P. 53-85.

[18] Бессе А. Многообразия Эйнштейна. 1, 2. М.: Мир, 1990.

[19] Takamatsu K., Watanabe Y. Classification of a conformally flat $K$-space // Tôhoku Math. J. 1972. V. 24. P. 435-440. 